\title{
Incidence of skin cancers in 3867 patients treated with narrow-band ultraviolet B phototherapy.
}

\begin{abstract}
Background: Narrow-band ultraviolet B (NB-UVB) phototherapy is a widely used treatment. Psoralen-UVA photochemotherapy (PUVA) increases skin cancer risk and some animal studies have raised the possibility of an increased risk with NB-UVB. The risk of skin cancer in humans following treatment with NB-UVB is unknown. Objectives: This current analysis forms part of an ongoing study ultimately aiming to define the long-term carcinogenic risk of NB-UVB treatment in humans. Methods: Details of all patients receiving NB-UVB treatment until 31/12/2002 in Tayside, Scotland, were accessed from a treatment database and linked to the Scottish Cancer Registry. Indirect standardization was used to compare skin cancer incidence in the study population with age and sex matched cancer registry data for the Tayside population. We also assessed the effect of NB-UVB exposure treatment numbers on the risk of developing skin cancer. Results: Of 4690 records reviewed, 4665 were suitable for analysis with 3886 records linked with the cancer registry and 3867 followed-up for at least 6 months before 31/12/02 (the date at which cancer registration was deemed to be complete). The median number of NB-UVB treatments was 29 with 352 patients receiving $\geq 100$ treatments. The study gave 24753 person-years of follow up. First skin cancers recorded in study patients were 27 basal cell carcinomas (BCC), seven squamous cell carcinomas (SCC) and six melanomas. No association was found between NB-UVB exposure alone (without PUVA) and any skin cancer. For NB-UVB and PUVA treated patients there was an association with BCC, with 27 BCCs found compared with 14.1 expected in the matched population. Conclusion: We found no significant association between NB-UVB treatment and BCC, SCC or melanoma. There was a small increase in BCCs amongst those also treated with PUVA. These reassuring results do not demonstrate the early increase in skin cancers that was found associated with PUVA treatment. However, cautious interpretation is required as the cohort contained relatively few patients who had a high treatment number and because the slow evolution of skin cancers may result in a delayed incidence peak. Ongoing risk assessment is therefore essential.
\end{abstract}

Keyword: Epidemiology; Psoriasis; Skin cancer; Ultraviolet B; Ultraviolet radiation. 